\title{
Blockchain: chaining digital health to a new era
}

\author{
Dongyuan Yun ${ }^{1}$, Wenben $C^{1}{ }^{1}$, Xiaohang $W^{1}{ }^{1}$, Daniel Shu Wei Ting ${ }^{1,2,3}$, Haotian Lin ${ }^{1,4}$ \\ ${ }^{1}$ State Key Laboratory of Ophthalmology, Zhongshan Ophthalmic Centre, Sun Yat-sen University, Guangzhou, China; ${ }^{2}$ Singapore Eye Research \\ Institute, Singapore National Eye Centre, Duke-NUS Medical School, National University of Singapore, Singapore; ${ }^{3}$ Department of Vitreo Retinal, \\ Moorfields Eye Hospital, London, UK; ${ }^{4}$ Centre of Precision Medicine, Sun Yat-sen University, Guangzhou, China \\ Correspondence to: Prof. Haotian Lin. State Key Laboratory of Ophthalmology, Zhongshan Ophthalmic Centre, Sun Yat-sen University, Jin Sui Road \\ 7\#, Guangzhou 510060, China. Email: gddlht@aliyun.com.
}

Submitted Mar 25, 2020. Accepted for publication May 25, 2020.

doi: $10.21037 / \mathrm{atm}-20-2854$

View this article at: http://dx.doi.org/10.21037/atm-20-2854

Blockchain technology, first known as Bitcoin, is an integration of distributed ledgers, asymmetric encryption, and hash algorithms to achieve decentralization, immutability, security and traceability (1). It eliminates intermediaries, transfers the trust from institutions to hard coding, and is considered the turning point to collapse the monopoly of data by returning control over information to individual owners (2). Ethereum, a symbol for advanced concoction, was empowered by smart contracts (3), which are coding programs that automatically self-execute the exchange of value once specific agreements are triggered. Most of the aforementioned applications are on public chains, which mean that everyone in the community has viewing permission. As the need increases in high-security industries such as pharmacies and healthcare, there has emerged a new type of blockchain, the consortium chain, with inspected members.

Blockchains can be utilized on a large scale in healthcare, where data silos caused by the centralized framework for data storage and the rigorous requirements for data security hinder information sharing, especially when billions of data points are generated by wearable devices and tremendous demands for data storage and utilization are created by artificial intelligence (AI). Furthermore, the advantages of blockchains over verifying the ownership of data and extending the spatial or temporal dimension $(4,5)$ also endow the concept of the blockchain with promising prospects in the healthcare industry. Guardtime partnered with the Estonian government to deploy a nationwide blockchain-based platform to validate patient identities and secure citizens' health records in a patient-centric way. This system ensured that patients could control their data and determine access permissions and was immune to malicious tampering, even with multiple stakeholders (6).

The involvement and interactions of more parties and nodes of clinical trials far from the main institutions produce the chance of human-induced error, whether accidental or malicious. The prototype designed by Wong et al. presented a blockchain-based solution for clinical trials to lock every alteration on the chain involving multiple parties under the inspection of regulators (7). Additionally, applicable scenarios due to immutability or traceability can be extended to pharmaceutical supply chain management, medical professional credentialing and licensing, and health insurance claims management $(8,9)$. Inspired by data monetization solutions to incentivize individual data sharing, companies such as Nebula Genomics have established blockchain-based data marketplaces to enable individuals to sell their biological data, including DNA sequences, to other stakeholders with digital rewards (10-12). Patients not only have control over their data but also have the right to share the profits generated by their data in the digital health ecosystem.

However, in view of the Gartner Hype Cycle, the blockchain is still in the stage between the "peak of inflated expectations" and the "trough of disillusionment" (13). For the healthcare industry, security and privacy are the main concerns. In general, the balance between transparency and confidentiality may be subtle when "everyone can see everything" on public chains, while further applications 
might concentrate on specific solutions utilizing consortium chains or private chains. Immutability is also a double-edged sword when patients expect to take their data off the chains. Furthermore, if all medical data are on the blockchain, it will become swollen as it does not fundamentally change how data are stored but rather changes how to retrieve and share data; thus, in most applications, data are stored outside the chains with cryptographic address pointers on the chains (14). Additionally, the application of a blockchain must be subject to elaborate consideration and reproductive testing by professionals. From 2016 to 2019, hackers obtained cryptocurrency of more than 80 million dollars by exploiting the bugs of smart contracts (15). Hence, before their widespread adoption, the feasibility of blockchains in healthcare remains to be tested on a larger scale.

Nevertheless, due to the evolution of technology, not just data can be distributed through blockchains but models and computing power can also be allocated, which implies that the mistrust about putting data on the chain may be decreased. With its fit-for-purpose, a blockchain-based pharmaceutical application, the Machine Learning Ledger Orchestration for Drug Discovery (MELLODDY) project, is expected to train AI models with numerous datasets without altering data storage or sacrificing data ownership. In addition, quantum computing might trigger fundamental changes in underlying cryptography (16), and future encrypted technology will be beyond what we can imagine.

Moreover, breaking down data silos utilizing blockchains might cause "technology silos" when data are locked into various chains without being connected to each other under general standards (8). Therefore, general standards for data sharing across blockchains are essential for releasing blockchains' dividends and promoting data sharing to the greatest extent. Sophisticated upstream and downstream facilities to back up extensive blockchain applications in healthcare are crucial. As data engines, they ultimately consume data to generate value and promote the development of the blockchain, in turn contributing to forming a closed-loop ecosystem (17). The sounder the standards among different chains and supporting facilities are, the clearer the blueprint of healthcare blockchain-based ecosystems will be realized.

The technology fusion of AI, big data mining, cloud computing, and supercomputers has unleashed the power of big data and technology and presented challenges in fit-for-blockchain scenarios. As mentioned above, in healthcare, the blockchain may be able to blend AI with other techniques to sufficiently address such data in the near future while breaking down data and technology silos. Although the blockchain is in its infancy, appropriate healthcare applications and research will spur the potential of the blockchain after it is securely tested by professionals and facilitate the establishment of the ecosystem.

\section{Acknowledgments}

Funding: This work was supported by grants from the National Key R \& D project (2018YFC0116500), the National Natural Science Foundation of China Cultivation Project (91546101, 81770967), the Science and Technology Planning Projects of Guangdong Province (2018B010109008), Guangdong Science and Technology Innovation Leading Talents (2017TX04R031), the Science and Technology Planning Projects of Guangdong Province (2019B030316012).

\section{Footnote}

Provenance and Peer Review: This article was commissioned by the Guest Editors (Haotian Lin and Limin Yu) for the series "Medical Artificial Intelligent Research" published in Annals of Translational Medicine. The article did not undergo external peer review.

Conflicts of Interest: All authors have completed the ICMJE uniform disclosure form (available at http://dx.doi. org/10.21037/atm-20-2854). The series "Medical Artificial Intelligent Research" was commissioned by the editorial office without any funding or sponsorship. HL served as the unpaid Guest Editor of the series. The other authors have no other conflicts of interest to declare.

Ethical Statement: The authors are accountable for all aspects of the work in ensuring that questions related to the accuracy or integrity of any part of the work are appropriately investigated and resolved.

Open Access Statement: This is an Open Access article distributed in accordance with the Creative Commons Attribution-NonCommercial-NoDerivs 4.0 International License (CC BY-NC-ND 4.0), which permits the noncommercial replication and distribution of the article with the strict proviso that no changes or edits are made and the original work is properly cited (including links to both the formal publication through the relevant DOI and the license). See: https://creativecommons.org/licenses/by-nc-nd/4.0/. 


\section{References}

1. Bai CA, Cordeiro J, Sarkis J. Blockchain technology: Business, strategy, the environment, and sustainability. Bus Strat Env 2020;29:321-2.

2. Schwab K, Davis N. Shaping the future of the fourth industrial revolution. Currency, 2018.

3. Agbo CC, Mahmoud QH, Eklund JM. Blockchain technology in healthcare: a systematic review. Healthcare (Basel) 2019;7:56.

4. Kamel Boulos MN, Wilson JT, Clauson KA. Geospatial blockchain: promises, challenges, and scenarios in health and healthcare. Int J Health Geogr 2018;17:25.

5. IBM Institute for Business Value. Healthcare rallies for blockchains: Keeping patients at the center. Somers: IBM, 2016.

6. Guardtime. Guardtime Industrial Blockchain. Available online: https://guardtime.com/

7. Wong DR, Bhattacharya S, Butte AJ. Prototype of running clinical trials in an untrustworthy environment using blockchain. Nat Commun 2019;10:917.

8. Mackey TK, Kuo TT, Gummadi B, et al. 'Fit-forpurpose?' - challenges and opportunities for applications of blockchain technology in the future of healthcare. BMC Med 2019;17:68.

Cite this article as: Yun D, Chen W, Wu X, Ting DSW, Lin H. Blockchain: chaining digital health to a new era. Ann Transl Med 2020;8(11):696. doi: 10.21037/atm-20-2854
9. Dimitrov DV. Blockchain applications for healthcare data management. Healthc Inform Res 2019;25:51-6.

10. Zhavoronkov A, Church G. The advent of human life data economics. Trends Mol Med 2019;25:566-70.

11. Nebula Genomics. San Francisco: Nebula Genomics. Available online: https://nebula.org/

12. Serono to pilot Nebula's blockchain for genomes. Nat Biotechnol 2019;37:706.

13. Gartner. Gartner hype cycle: Interpreting technology hype. 2018. Available online: https://www.gartner.com/ en/research/methodologies/gartner-hype-cycle

14. Giordanengo A. Possible usages of smart contracts (blockchain) in healthcare and why no one is using them. Stud Health Technol Inform 2019;264:596-600.

15. Douthwaite A. Just how smart are Smart Contracts? 2017. Available online: https://www.csoonline.com/ article/3236054/just-how-smart-are-smart-contracts. html

16. Fedorov AK, Kiktenko EO, Lvovsky AI. Quantum computers put blockchain security at risk. Nature 2018;563:465-7.

17. Krittanawong C, Rogers AJ, Aydar M, et al. Integrating blockchain technology with artificial intelligence for cardiovascular medicine. Nat Rev Cardiol 2020;17:1-3. 